\title{
- Pourquoi les Agences de l'Eau françaises servent-elles de modèle à la politique communautaire de l'environnement ?
}

— Why are the french "Agences de l'Eau" a model for the environment european policy ? —

\author{
par Robert Galley \\ Ancien ministre, Député de l'Aube, Président du Comité de Bassin Seine-Normandie
}

\section{Les Agences de l'Eau françaises offrent un fonctionnement tout à fait satisfaisant. Ne pourraient-elles constituer un exemple pour les autres pays de I'Union européenne?}

Après avoir inventé la roue, l'homme essaya de la perfectionner. Il en construisit toutes sortes : des roues heptagonales, des hexétagonales et même des kilogonales. Mais aucune ne fonctionnait aussi bien que la roue d'origine qui, elle, possédait un secret : elle ne comportait aucun côté droit et même, à dire vrai, aucun côté. Mais les experts locaux de cette époque n'en croyaient rien et s'évertuaient à vouloir améliorer la forme des roues...

Les Agences de l'Eau françaises fonctionnent un peu comme des roues, ou des vis d'Archimède, si l'on préfère. Elles s'inspirent d'un principe de circularité entre les moyens financiers et les finalités techniques de leurs programmes d'action, entre les usagers qui payent des redevances et les bénéficiaires des aides financières qui stimulent des réalisations et des progrès de fonctionnement.

Certes la roue peut être plus grande, plus rapide, mais elle ne peut pas être plus ronde que sa forme actuelle. Que les experts en évaluation linéaire s'en persuadent les approximations qu'ils suggèrent sont des régressions les développements qui suivent en détaillent les raisons.

\section{EINTRODUCTION}

Au moment où la France s'apprête à organiser une grande conférence internationale sur l'eau (" l'eau et le développement durable ", Paris, 19-21 mars 1998) et où l'Union Européenne se prépare à adopter une directivecadre sur l'eau, les regards se tournent vers un instrument particulier de la politique de l'environnement: les agences financières de bassin, récemment nommées Agences de l’Eau.

Les Agences de l'Eau françaises apparaissent être l'un des instruments économiques au monde, réellement opérationnel depuis maintenant trente ans, qui réussit à optimiser la protection de l'environnement.

Adopté récemment par le Conseil de I'Union Européenne, le cinquième programme communautaire d'action pour l'environnement prévoit un recours croissant aux instruments économiques. C'est surtout dans le domaine des ressources en eau et pour la lutte contre leur pollution que de tels instruments existent. Ils sont utilisés en France depuis 1967, l'un des premiers pays à avoir mis en œuvre le principe Pollueur-Payeur, associé à l'exercice de la solidarité de bassin.

Les avantages des Agences de l'Eau sont triples : ce sont des institutions économiques (II), elles font appel à la solidarité des usagers au moyen de décisions démocratiques déconcentrées (III), elles contribuent puissamment à la protection de l'environnement par l'affectation directe de leurs ressources financières à des projets spéci-

In France, the principle "who pollutes, pays" has been applied since 1967. The "Agences de l'Eau" are an economic instrument for applying this principle and they actively participate in the environment protection. Their actions favour industries and citizens initiatives. 
fiques, dans le respect de la mise en place d'un grand marché communautaire (IV).

\section{II ${ }^{-1}$ LES AGENCES DE L'EAU, INSTRUMENT ÉCONOMIQUE DE PROTECTION DE L'ENVIRONNEMENT}

Les redevances perçues par les Agences de l'Eau, audelà de leur importance financière, constituent un signal économique clair destiné aux divers usagers de l'eau. Celui qui prélève et consomme de l'eau la paye, celui qui pollue paye également le prix de son épuration.

Grâce aux redevances, des programmes de mesures en faveur d'une protection de l'environnement peuvent être élaborés sans recourir au budget de l'Etat. Chaque bassin optimise ses recettes et ses programmes au niveau de protection le plus élevé possible et dans un souci de compétitivité industrielle et agricole accrue. Il s'efforce d'assurer aux usagers domestiques une alimentation en eau de la meilleure qualité possible et prend soin de vérifier leur capacité à payer le prix de l'eau dont environ un cinquième est constitué des taxes et redevances.

Pour tous ceux qui ne payent pas directement l'eau, ces redevances ne sont jamais assez chères. Pour tous ceux qui au contraire la payent, son prix est toujours trop élevé. C'est en cela que le niveau de redevances décidé volontairement par chaque Comité de bassin pour une période donnée (en ce moment 1997-2001) constitue un optimum économique qu'aucune mesure centrale autoritaire n'a de chance de pouvoir mieux approcher.

\section{U UN BEL EXEMPLE DE DÉMOCRATIE : LA MISE EN GEUVRE DES ACTIONS DES AGENCES DE L'EAU REPOSE SUR LES USAGERS}

Le processus décisionnel des Agences de l'Eau repose largement sur les Comités de Bassin où les usagers représentent $80 \%$ des membres. Les Comités de Bassin élaborent les programmes quinquennaux d'activités et votent les taux de redevances qui les équilibrent. Ces dispositions s'insèrent particulièrement bien dans le cadre institutionnel et politique communautaire. Celui-ci est en effet de plus en plus favorable aux actions volontaires des citoyens et des industriels. Il cherche par ailleurs à faire progresser l'idée de subsidiarité largement maîtrisée par les Agences de l'Eau.

Tous les usagers sont traités avec équité c'est-à-dire en fonction de leur seul rattachement naturel à la ressource hydraulique disponible, tant en quantité qu'en exigence de qualité. En intégrant ainsi toutes les déséconomies externes de production (la surconsommation, la pollution rejetée), les Agences de l'Eau se situent dans la droite ligne des principes fondamentaux du droit communautaire hostile à toute discrimination.

\section{IV $\square$ LES AGENCES DE L'EAU, INSTRUMENT DE L'AVENIR ET DU DÉVELOPPEMENT DURABLE}

La politique communautaire de concurrence s'efforce d'établir l'équilibre du fonctionnement du marché en tenant compte proportionnellement de toute atteinte à la concurrence due au non-respect des objectifs environnementaux prescrits.

Cette règle de proportionnalité est assurée par les Agences de l'Eau. En redistribuant les redevances perçues, elles contribuent à la protection de l'environnement dont le traité sur l'Union Européenne a fait l'objet de l'une de ses politiques fondamentales, la dotant même d'une sorte de primauté sur toutes les autres.

Le cinquième programme communautaire d'action place d'ailleurs la gestion des ressources en eau au rang des questions particulièrement graves qui touchent l'ensemble de la communauté, considérant qu'il est essentiel d'en assurer durablement le développement.

Le dispositif des Agences de l'Eau est de plus très largement compatible avec l'intégration du grand marché puisqu'il satisfait à l'objectif de transparence. Loin de fausser la concurrence, il allège le désavantage compétitif que constitue pour une entreprise un investissement en faveur de la protection de l'environnement.

Les programmes des six Agences de l'Eau Françaises sont largement conformes à la politique communautaire de l'environnement. Leurs actions sont proportionnelles, ni moins ni plus, aux objectifs ambitieux de cette politique. En mettant en œuvre le principe pollueur-payeur, elles font appel à des engagements volontaires des usagers, quels qu'ils soient, dans un esprit de compréhension partagée des enjeux et non de réaction à des dispositions autoritaires.

L'Agence de l'Eau Seine-Normandie est la plus importante des six Agences de l'Eau Françaises. Elle s'étend des sources de la Seine en Côte d'Or jusqu'au Couesnon qui sépare la Normandie de la Bretagne (et qui mit dans sa folie - le Mont-Saint-Michel en Normandie!). Elle s'étend même plus à l'ouest jusqu'aux communes de Saint-Pierre et de Miquelon, tout près de Terre-Neuve, Canada.

L'Agence Seine-Normandie est jumelée à l'Agence de l'Environnement de la Tamise (Environment Agency, Thames Region), à l'Office Bavarois de l'Eau (Bayerisches Staatministerium für Landesentwicklung und Umweltfragen), au service Voyer et de gestion des eaux du Hainaut (Belgique), à la confédération hydraulique du bassin du Guadalquivir (Confederacion Hidrografica del Guadalquivir), à l'office des eaux de Hongrie (Office National de l'Eau). Elle développe des réflexions sur l'avenir de la politique de l'eau en Europe et dans le monde au sein du Forum Européen pour l'Economie et l'Eau, également grâce aux travaux de l'Académie de l'Eau et en relation avec le Conseil Mondial de l'Eau. 